\title{
BMJ Open Efficacy and cost-effectiveness of Stem Cell injections for symptomatic relief and strUctural improvement in people with Tibiofemoral knee OsteoaRthritis: protocol for a randomised placebo- controlled trial (the SCUlpTOR trial)
}

\author{
Xiaoqian Liu (1) , ${ }^{1,2}$ Sarah Robbins, ${ }^{1,2}$ Xia Wang (1) , ,2 Sonika Virk, ${ }^{1,2}$ \\ Karen Schuck, ${ }^{1,2}$ Leticia A Deveza, ${ }^{1,2}$ Win Min Oo, ${ }^{1,2}$ Kirsty Carmichael, ${ }^{3}$ \\ Benny Antony, ${ }^{3}$ Felix Eckstein, ${ }^{4,5}$ Wolfgang Wirth, ${ }^{4,5}$ Christopher Little, ${ }^{6}$ \\ James Linklater, ${ }^{7}$ Anthony Harris (1) , ${ }^{8}$ David Humphries, ${ }^{9}$ R O'Connell, ${ }^{10}$ \\ Gillian Heller, ${ }^{10}$ Thomas Buttel, ${ }^{11}$ Stefan Lohmander (D) , ${ }^{12}$ Changhai Ding, ${ }^{3,13}$ \\ David J Hunter (1) 1,2,13
}

To cite: Liu X, Robbins S, Wang $X$, et al. Efficacy and cost-effectiveness of Stem Cell injections for symptomatic relief and strUctural improvement in people with Tibiofemoral knee 0steoaRthritis: protocol for a randomised placebo-controlled trial (the SCUIpTOR trial). BMJ Open 2021;11:e056382. doi:10.1136/ bmjopen-2021-056382

- Prepublication history and additional supplemental material for this paper are available online. To view these files, please visit the journal online (http://dx.doi.org/10.1136/ bmjopen-2021-056382)

Received 15 August 2021 Accepted 08 November 2021

Check for updates

(c) Author(s) (or their employer(s)) 2021. Re-use permitted under CC BY-NC. No commercial re-use. See rights and permissions. Published by BMJ.

For numbered affiliations see end of article.

Correspondence to Professor David J Hunter; david.hunter@sydney.edu.au

\section{ABSTRACT}

Introduction Knee osteoarthritis (KOA) is a highly prevalent disabling joint disease. Intra-articular stem cell therapy is increasingly being used for treating KOA with little high-quality evidence to support its use. The aim of this study is to investigate the efficacy, safety and cost-effectiveness of allogeneic mesenchymal stem cells (Cymerus MSCs) for treating symptomatic tibiofemoral KOA and improving knee structure over 24 months. Methods and analysis The Stem Cell injections for symptomatic relief and strUctural improvement in people with Tibiofemoral knee 0steoaRthritis study is a phase III, multi-centre, parallel, superiority, randomised, doubleblind, placebo-controlled trial, which will be conducted in Sydney and Hobart, Australia. 440 participants (220 per arm) aged over 40 years with painful KOA and mild to moderate structural change on X-ray (Kellgren and Lawrence grade 2 or 3 ) with medial minimum joint space width between 1 and $4 \mathrm{~mm}$ in the study knee will be recruited from the community and randomly allocated to receive either intra-articular MSCs or saline at baseline, week 3 and week 52 . The coprimary outcomes will be the proportion of participants achieving patientacceptable symptom state for knee pain at 24 months and quantitative central medial femorotibial compartment cartilage thickness change from baseline to 24 months. Main secondary outcomes include change in knee pain, Patient Global Assessment, physical function, quality of life and other structural changes. Additional data for cost-effectiveness analysis will also be recorded. Adverse events will be monitored throughout the study. The primary analysis will be conducted using modified intention-totreat.

Ethics and dissemination This protocol has been approved by The University of Sydney (USYD) Human Research Ethics Committee (HREC) \#: 2020/119 and The

\section{Strengths and limitations of this study}

- The Stem Cell injections for symptomatic relief and strUctural improvement in people with Tibiofemoral knee 0steoaRthritis trial is an investigator-initiated, multi-centre, 2-year randomised clinical trial with a large sample size and robust methodology assessing both clinical and radiographic endpoints, which will provide high-quality evidence in examining the efficacy of allogeneic mesenchymal stem cell (MSC) injections for knee osteoarthritis (KOA).

- The trial will use online recruitment and data capture to minimise face-to-face clinic visits, which will increase recruitment efficiency; additionally, people with KOA have been involved in the study design, which will help identify logistical issues to facilitate adherence to the study.

- The trial will only include participants with tibiofemoral KOA, which may limit generalisability to those with $\mathrm{KOA}$ in other knee compartments in clinical practice.

- The trial will only include treatment with a single specific allogeneic MSC source/type, dose and treatment schedule, which may limit generalisability to other cell types or dosing regimens.

- In order to facilitate comparison with other injection trials, normal saline was chosen as the placebo treatment; we acknowledge that the carrier media of the active and placebo group is different with cryoprotectant and electrolytes for the stem cell.

University of Tasmania (UTAS) HREC \#: H0021868. All participants will be required to provide informed consent. Dissemination will occur through conferences, social media, and scientific publications. 
Trial registration numbers Australian New Zealand Clinical Trials Registry (ACTRN12620000870954); U1111-1234-4897.

\section{INTRODUCTION}

\section{Background and rationale}

Osteoarthritis (OA) is a highly prevalent and progressive joint disease associated with inflammation and major structural changes of the affected joint. ${ }^{2}$ Knee osteoarthritis (KOA) accounts for $85 \%$ of the burden of OA worldwide and affects $19 \%$ of people aged over $45 \%$ and $37 \%$ of people aged over 60 years of age. ${ }^{3-5}$ KOA causes substantial pain and physical dysfunction, ultimately impairing quality-of-life and is ranked as the eleventh highest contributor to global disability. ${ }^{6}$ The average total expense per KOA patient per annum is over US\$15000, totalling over US\$34 billion in healthcare expenditure. ${ }^{7}$ The healthcare costs of KOA are projected to double by 2040 given population ageing and escalation in obesity, driving an increase in $\mathrm{KOA}^{8}{ }^{8}$

As a disease of the whole joint, KOA requires longterm management with various treatment options over the course of the disease. However, the current treatment modalities (eg, exercise therapy, paracetamol, nonsteroidal anti-inflammatory drugs, intra-articular steroids) do not target structural pathology, and are either only modestly effective in alleviating symptoms, have significant potential side effects or both. ${ }^{910}$ Therefore, there is a need for a sustainable long-term effective treatment focusing on mitigating the epidemic of OA, modifying its structural progression and symptomatic consequences. ${ }^{11}$

Most recently, stem cells have emerged as an intraarticular option for KOA, although the quality of the current evidence to support its use is low due to poor trial design (eg, inadequate blinding, biases, small studies). ${ }^{12}$ The current stem-cell market for KOA is rapidly expanding with $80 \%$ of patients claiming 'symptomatic improvement'. ${ }^{13}$ Given the considerable cost of treatment with an average US $\$ 5000$ per injection ${ }^{13}$ and very limited scientific evidence of efficacy and safety, high-quality evidence from randomised clinical trials is necessary to define the role of stem cell therapies in the treatment of KOA.

As multipotent precursor cells, mesenchymal stem cells (MSCs) have been suggested to be effective through their release of trophic factors that modulate inflammation and recruit and stimulate resident cells to enhance repair. ${ }^{14}$ This effect has been assessed both preclinically and clinically in a pilot trial, which showed an increase in the articular cartilage volume assessed by MRI. ${ }^{15}$ Other pilot trials or phase II studies aslo showed clinically significant pain and functional improvement without observed serious adverse events (AEs). ${ }^{16-19}$ The studies indicated that repeated intra-articular injections of MSCs achieved more consistent OA stabilisation than a single injection. ${ }^{16}$ MSCs can be isolated from numerous tissues but those for intra-articular injection are presently derived mainly from autologous adipose tissue or bone marrow. ${ }^{2021}$ Given the additional procedure for cell harvesting and the potential presence of comorbidities in the preparation of autologous stem cells, the use of potential off-the-shelf commercial preparations of allogeneic MSCs from healthy donors may reduce the overall cost of cell therapies, while maintaining an accurate quality control. ${ }^{22}$

Cymerus MSCs, produced from induced pluripotent stem cells (iPSCs) through the mesenchymoangioblast pathway, have surface markers and trilineage differentiation, which meet International Society for Cellular Therapy criteria for defining multipotent MSCs, ${ }^{23} 24$ and have been shown to be safe and well-tolerated in humans. ${ }^{25}$ The iPSCs were derived from CD34-enriched peripheral blood mononuclear cells using an episomal plasmidbased, transgene-free, viral-free and feeder layer-free reprogramming procedure. Although in vivo study of intra-myocardial administration of Cymerus MSCs showed a pro-angiogenic secretory profile with upregulation of pro-angiogenic factors and downregulation of metalloproteinases, ${ }^{26}$ this is the first time that Cymerus MSCs is being used intra-articularly for OA. Therefore, the aim of this study is to investigate the efficacy, safety and costeffectiveness of Cymerus MSCs for treating symptomatic tibiofemoral KOA and improving knee joint structure in comparison to placebo over 24 months.

\section{Objectives}

The primary objective is to determine the efficacy of intra-articular allogeneic MSCs on the proportion of participants achieving patient-acceptable symptom state for knee pain and the reduction in loss of central medial femorotibial cartilage thickness compared with placebo (saline) over 24 months in people with symptomatic tibiofemoral KOA. The secondary objectives are to assess the efficacy on other clinical and structural outcomes as well as the safety and cost-effectiveness of MSCs therapy.

\section{METHODS AND ANALYSIS \\ Study design}

The Stem Cell injections for symptomatic relief and strUctural improvement in people with Tibiofemoral knee OsteoaRthritis (SCUlpTOR) trial is designed as a parallel, superiority, randomised, double-blind, placebocontrolled, two-arm clinical trial with 1:1 allocation ratio. The protocol is described using the Standard Protocol Items: Recommendations for Interventional Trials guidelines on standard protocol items for clinical trials and the results will be reported using the Consolidated Standards of Reporting Trials statement (online supplemental file 1 full study protocol version 8, 18 June 2021).

\section{Study setting}

The SCUlpTOR trial will be conducted in Sydney and Hobart and will include two research institutes and an imaging centre. The recruitment rate at each site will be independent of each other:

1. Sydney. 
i. Institute of Bone and Joint Research (IBJR), Kolling Institute of Medical Research, The University of Sydney (administrative procedures).

ii. Castlereagh Imaging, St Leonards (face-to-face study procedures).

2. Hobart.

i. Menzies Institute for Medical Research, University of Tasmania (administrative and face-to-face study procedures).

The IBJR will be the coordinating centre for this trial.

\section{Participant recruitment and eligibility criteria}

Four hundred and forty participants (220 per arm) with symptomatic KOA will be recruited from the community and research volunteer databases. The recruitment strategies will include: (a) mailed/emailed advertisements to the research volunteer databases; (b) posters/flyers placed on medical practices and community areas or newsletters/e-newsletters in the universities and institutes; (c) advertisements/postings on social media networks (eg, Facebook, Twitter) and research institute websites; (d) local and major newspaper advertisements or newsletter listings; (e) radio or TV interviews; (f) clinical trial recruitment companies; $(\mathrm{g})$ community-based events.

Participants will be eligible for the study if they meet all the inclusion criteria listed below:

1. Ability and willingness to participate and complete the study.

2. Functional English, internet access and an active email account.

3. $\geq 40$ years old, male or female, Australian citizen or permanent resident having a valid medicare account for cost-effectiveness analysis.

4. Presence of knee pain for at least half of the days in the previous month (the most painful knee will be considered as the study knee).

5. Average pain intensity $\geq 40$ and $\leq 90$ out of 100 on a Visual Analogue Scale (VAS) in the study knee over the last week prior to the online screening and baseline surveys.

6. Kellgren and Lawrence grade (KLG) two or three and medial tibiofemoral minimum joint space width between 1 and $4 \mathrm{~mm}$ of the study knee based on fixed-flexion posteroanterior (PA) knee radiograph using Synaflexer, which will be assessed by a trained rheumatologist with experience in KOA research.

7. KOA defined by the American College of Rheumatology (ACR) criteria using medical history, patient-reported symptoms/signs and radiographic findings: ${ }^{27}$ (a) knee pain on most days; (b) osteophytes on X-ray and (c) one of the three following criteria: age $>50$ years, less than $30 \mathrm{~min}$ of morning stiffness and crepitus on active motion.

8. Willingness to undergo a new knee X-ray and MRI.

9. Willingness to stop or maintain a routine (ie, on the same dosage and frequency) of conservative treatments (eg, physiotherapy, exercise, knee brace, oral supplements) for the duration of the study.
10. Willingness to stop or minimise the use of nonsteroidal anti-inflammatory drugs (NSAIDs) and other analgesics (except paracetamol for rescue pain relief) for the duration of the study.

11. Willingness to undergo a 1-week medication washout (for all pain medications) before each pain assessment survey at baseline, 3, 6, 9, 12, 15, 18, 21 and 24 months.

12. Willingness to avoid a new treatment for KOA during the study.

13. Willingness and ability to travel to the study visits at either Castlereagh Imaging (St Leonards and Cremorne in Sydney) or Menzies Institute (Hobart) and Qscan Radiology (North Hobart).

Participants will be excluded if they meet any of the following criteria:

1. Incomplete online screening surveys or nonresponders after completing their online screening survey but before being enrolled in the study.

2. Women who are pregnant or breast feeding, or women of childbearing potential not willing to use contraceptive methods for the duration of the study.

3. Radiographic evidence of predominant lateral tibiofemoral or patellofemoral disease based on the severity of joint space narrowing in each compartment.

4. Bilateral symptomatic KOA if the patient-reported pain intensity in the contralateral knee is $\geq 30$ on a 0-100 VAS.

5. Significant injury in the study knee that led to substantial loss of function or surgeries in the past 6 months (eg, fracture, ligament rupture, joint dislocation, trauma, laceration or nerve damage).

6. Surgery on the study knee in the past 12 months or expected joint surgery for the study knee in the next 24 months.

7. Prior knee joint replacement or high tibial osteotomy in the study knee.

8. History of crystalline (eg, gout, calcium pyrophosphate deposition disease), autoimmune arthritis (eg, rheumatoid arthritis, psoriatic arthritis, systemic lupus erythematosus, ankylosing spondylitis), haemochromatosis or fibromyalgia. Except for the following conditions:

i. Participants diagnosed with gout are eligible for the study as long as the condition is being appropriately treated and they have not experienced flare-ups for at least 12 months.

ii. Participants diagnosed with haemochromatosis but with normal iron levels for at least 12 months are eligible for the study.

9. Signs of acute knee joint inflammation (ie, red, swollen and hot) and/or abnormal synovial fluid suggestive of crystals or infection.

10. Any painful muscular or neurological condition of the lower limb that, in the opinion of the investigator, is the main contributor to the pain and/or loss of function in the study knee which may interfere with the self-reported assessment (eg, fracture, ligament 
rupture, bursitis, tendinitis, hypermobility syndrome, joint infection, patellofemoral pain syndrome, diabetic neuropathy, pain referred from the back, pain following hip, knee or ankle trauma or surgery).

11. Cancer or other tumour-like lesions, except for skin cancer (non-melanoma, removed and not active for at least 3 years).

12. Immunosuppression or acute infective processes.

13. Intra-articular hyaluronic acid injection in the study knee in the past 6 months; intra-articular steroid injections in any joint in the past 6 months; intraarticular autologous blood product or stem cell injection in the study knee in the past 12 months.

14. Regularly taking centrally acting analgesics (eg, opioid analgesics, duloxetine and pregabalin).

15. Participation in other clinical trial and/or treatment received with any investigational agent within 30 days before enrolment.

16. Any unstable concurrent clinically significant acute, chronic medical conditions or abnormal laboratory findings that, in the judgement of the investigator, would jeopardise the safety of the patient, interfere with the objectives of the protocol, or affect the participants' compliance with the study requirements.

17. Needle phobia.

18. Contraindication to MRI including but not limited to a pacemaker, metal sutures, presence of shrapnel, or claustrophobia and/or inability to fit into the MRI knee coil.

\section{Interventions}

The active group will receive three intra-articular knee injections of Cymerus MSCs at baseline, week 3 and week 52. Each injection will consist of $2.5 \times 10^{7}$ cell cultureexpanded allogeneic MSCs, which are suspended in $5 \mathrm{~mL}$ excipient solution containing multiple electrolytes injection with $10 \%$ human serum albumin and $2.5 \%$ dimethyl sulfoxide as a non-toxic cryoprotectant. The placebo group will receive three intra-articular knee injections of saline containing $0.9 \%$ sodium chloride at the same timeframe. The rationale for choosing normal saline as the comparator as distinct from excipient is to facilitate comparison with other injection trials where the magnitude of effect from normal saline is known. The intra-articular knee injections will be guided by ultrasound using one of the three methods: (a) lateral suprapatellar approach (effusion present); (b) patellofemoral approach (effusion absent) or (c) medial infrapatellar approach (effusion absent).

Cymerus MSCs (CYP-004) will be provided by Cynata Therapeutics (Carlton, Victoria, Australia) and manufactured by Waisman Biomanufacturing (Madison, Wisconsin, USA) according to the US current Good Manufacturing Practice standards. The Cymerus MSCs will be produced in batches according to the recruitment schedule due to the limited shelf life of 2 years from the date of manufacture. All batches of Cymerus MSCs product are derived from the same donor/master cell bank and subjected to an extensive series of in-process and release tests to optimise safety and batch to batch reproducibility. The MSCs will be stored in the vapour phase of liquid nitrogen, under temperature-controlled conditions at each research institute site (ie, Kolling and Menzies).

\section{Randomisation, allocation and blinding}

Eligible participants who consent to take part in the study will be assigned to either active or placebo group with a 1:1 allocation rate as per computer-generated randomisation scheduled using random permuted block sizes and stratified by study sites (Sydney and Hobart) and radiographic disease severity (ie, KLG 2 vs 3$)$.

The treatment allocation will only be available to the unblinded researcher using Research Electronic Data Capture (REDCap) randomisation module. The injecting doctors, study coordinators, study physician, imaging readers and study statistician will remain blinded until the main results are analysed. The study participants will be blinded to group allocation until the end of the study after the final assessments at 24 months. There will be immediate unblinding procedures available where there is a need due to medical issues. The assigned study intervention may need to be modified or discontinued in the case of AEs. The study coordinator will modify the study intervention in agreement with the Principal Investigators. Participants undergoing modifications will be retained in the trial.

\section{Concomitant and excluded medications and care}

Participants will continue to take medications for other health conditions as usual. Participants who are on a stable dose of supplements, physiotherapy or other conservative treatments for KOA will be asked to either stop or maintain their existing treatment regimens for the duration of the trial. The use of oral or topical NSAIDs and other analgesics for KOA will be either discontinued or maintained at their lowest dosage for the duration of the trial. The following medications and interventions will be prohibited during the study: (a) use of centrally acting analgesics (eg, opioids, duloxetine and pregabalin); (b) investigational products from another clinical trial; (c) intra-articular injections of any other agents; (d) surgery in the study knee; (e) any new treatment for KOA. Paracetamol (up to $3000 \mathrm{mg} /$ day) will be allowed to use as rescue pain relief during the study.

Concomitant and excluded medications mentioned above will be monitored using fortnightly and monthly surveys. Participants will be asked to undergo a 1 week pain medication wash-out before each pain assessment survey at baseline, 3, 6, 9, 12, 15, 18, 21 and 24 months to ensure the self-reported pain intensity is accurate. There will be at least 7 days gap between the treatment injections and any dose of vaccines (ie, COVID-19 vaccine). 


\section{Outcome measures}

The descriptions of primary, secondary and exploratory outcome measures are as below:

1. Patient-Acceptable Symptom State (PASS): PASS is defined as the value beyond which patients consider themselves well. The patient-reported knee pain intensity will be measured using VAS from 0 (no pain) to 100 (worst pain possible) with the question 'How much pain in your knee did you experience on average during knee movement while performing daily activities over the past week?'. The PASS threshold for pain intensity in people with KOA is less than $32 \mathrm{~mm}$ on the $0-100 \mathrm{~mm}$ VAS. $^{28}$

2. Central medial femorotibial compartment (cMFTC) cartilage thickness: the cMFTC cartilage thickness will be computed from segmentations of the weightbearing femorotibial cartilages performed by manually drawing disarticulation contours around the cartilage edges, section by section in all MRI slices depicting the cartilage of the study knee. ${ }^{29}$ The MRI will use a 3.0T whole-body system with dedicated extremity coil and a fat suppressed, 3D double echo at steady state (DESS) sequence.

3. Wong-Baker FACES Pain Rating Scale (WBS): the WBS is used to measure pain on a numerical scale $(0,2,4,6,8$ or 10$)$ with six faces, where the patient marked the face that better described the pain intensity. The number 0 and a smiling face denote no pain, while the number 10 and a crying face denote the most severe pain. ${ }^{30}$

4. Patient Global Assessment (PGA): PGA will be assessed using the question 'Considering all the ways your knee osteoarthritis affects you, how have you been during the past week?' along with a 0-100 VAS where 0 is very well and 100 is very poor.

5. Knee injury and Osteoarthritis Outcome Score (KOOS): the KOOS is a knee-specific instrument, developed to assess the patients' opinion about their knee and associated problems, which holds 42 items in five separately scored subscales, ${ }^{31}$ as detailed below:

i. Pain: nine questions regarding knee pain in the last week during various positions or movements contribute to the score, which ranges from 0 to 100 with lower scores indicating worse pain.

ii. Other symptoms: seven questions regarding knee symptoms over the last week contribute to the score, which ranges from 0 to 100 with lower scores indicating worse symptoms.

iii. Function in daily living (ADL): seventeen questions regarding the degree of difficulty performing daily activities over the last week contribute to the score which ranges from 0 to 100 with lower scores indicating worse function.

iv. Function in sport and recreation: five questions regarding the degree of difficulty performing sports and recreation activities over the last week contribute to the score which ranges from 0 to 100 with lower scores indicating worse function. v. Knee-related quality of life: four questions regarding the knee-related quality of life over the last week contribute to the score which ranges from 0 to 100 with lower scores indicating worse quality of life.

6. Physical Activity Scale for the Elderly (PASE): the PASE is a self-report measure designed to capture and assess occupational, household and leisure activities typically performed by older adults. Twelve questions regarding levels of physical activity over the past week contributes to the score, which ranges from 0 to 793 with higher scores indicating greater levels of physical activity. $^{32}$

7. Assessment of Quality of Life-Eight Dimensions (AQoL-8D): the AQoL-8D is a 35-item health utility instrument, consisting of two super dimensions of physical and mental health or eight dimensions: independent living, pain, senses, mental health, happiness, coping, relationships and self-worth. Thirty-five questions of eight dimensions regarding physical and psychosocial quality of life over the past week contribute to the score, which ranges from 0 to 100 , with higher scores indicating better quality of life. ${ }^{33}$

8. Cartilage thickness in other femorotibial plates and subregions: Cartilage thickness of total femorotibial, lateral femorotibial, medial femorotibial, medial tibial, medial femoral, lateral tibial and lateral femoral will be measured using the same method as the cMFTC cartilage thickness described above.

9. MRI Osteoarthritis Knee Score (MOAKS): the MOAKS instrument is an MRI semi-quantitative scoring of KOA, which was developed and tested on images obtained on a 3.0T MRI system with a dedicated peripheral knee coil. ${ }^{34}$ The subscores of knee structural changes are detailed as below:

i. Change in the number of areas with worsening in cartilage thickness categorised as $0,1,2$, or $\geq 3$.

ii. Worsening in osteophytes scored in each of the 12 locations according to size and categorised as yes or no.

iii. Change in bone marrow lesions (BMLs) scored based on the standardised regions ranging from 0 to 3 . Medial tibial and medial femoral condyle region BML scores will be added using categorical scoring (range 0-3 per region).

iv. Worsening in meniscal morphology features scored on medial and lateral meniscus for the anterior, body and posterior horn and categorised as yes or no.

v. Change in whole knee effusion (effusion-synovitis) categorised as 'worsen', 'no change' or 'improve'.

vi. Change in infra-patellar fat pad synovitis (Hoffa's synovitis) categorised as 'worsen', 'no change' or 'improve'.

10. Global rating of change (GRC): the GRC scales are designed to quantify a patient's improvement or deterioration over time to determine the effect of an intervention. ${ }^{35}$ The GRC for pain, function and overall 
will be assessed using the question 'Which option best represents the change in pain/change in function/overall change in your knee since you began the study?', scored using a 5-point Likert scale ranging from much better to much worse.

11. Treatment satisfaction: participants' opinion of satisfaction will be assessed using a 'yes/no' question 'Taking into account all the activities you have during your daily life, your level of pain, and also your functional impairment, do you consider that your current state is satisfactory?'. For those who answer 'no', their opinion of treatment failure will be assessed using a 'yes/no' question 'Would you consider your current state as being so unsatisfactory that you think the treatment has failed?'

12. Quality-adjusted life year (QALY): the QALY is a multidimensional measure of health outcome that encompasses both quality-of-life and quantity-of-life (survival) gains, which will be calculated by multiplying life years by the index of utility derived from the AQoL-8D on the QALY scale ranging from 0 (equivalent of being dead) to 1 (full health) ${ }^{36}$

13. Cartilage T2 relaxation time estimates: the T2 relaxation time will be assessed from the DESS MRI for the same regions of interest as for cartilage morphometry.

14. Cost-effectiveness: the cost-effectiveness analysis will be performed using a combination of the Medication Benefits Scheme/Pharmaceutical Benefits Scheme (MBS/PBS) data extracted for the study period and the monthly healthcare usage surveys.

15. Consumption of rescue medication: the consumption of paracetamol and other pain medications will be monitored by inspection of fortnightly surveys, which will be reported by participants.

16. Individual patient placebo response: baseline Multidimensional Psychological Questionnaire (MPsQ) modules will be used to assess the placebo response, ${ }^{37} 38$ which is a self-reported questionnaire using a 5-point scale ranging from 1 (strongly disagree) to 5 (strongly agree) contributing to the evaluation of individual patient response to placebo by assessing participants' basic personality traits, expectation traits and perception.

17. Blinding success: will be measured by asking which treatment participants believe they received. The injecting doctors and blinded assessors will also be asked which treatment they believe was given to the participants.

18. Treatment adherence will be reported as the number of injections administered.

19. AEs will be assessed at each study visit and by inspection of monthly surveys.

\section{Trial endpoints}

The primary endpoints will be:

1. The proportion of participants reaching PASS threshold for pain intensity (VAS $\leq 32$ ) at 24 months.
2. Change in cMFTC cartilage thickness from baseline to 24 months.

The secondary endpoints are:

1. Change in knee pain intensity from baseline to $3,6,9$, $12,15,18,21$ and 24 months using VAS and WBS.

2. Change in PGA from baseline to 3, 6, 12 and 24 months.

3. Change in KOOS from baseline to 3, 6, 12 and 24 months.

4. Change in PASE from baseline to 3, 6, 12 and 24 months.

5. Change in AQoL-8D from baseline to 3, 6, 12 and 24 months.

6. Change in cartilage thickness (quantitative) in other femorotibial plates and subregions from baseline to 24 months.

7. Change in structural knee features assessed by MOAKS (semi-quantitative) from baseline to 24 months.

8. Change in cartilage T2 relaxation time estimates: the T2 relaxation time will be assessed from the DESS MRI for the same regions of interest as for cartilage morphometry.

The exploratory endpoints include: (a) GRC for pain, function and overall at 3, 6, 12 and 24 months; (b) treatment satisfaction at 24 months; (c) QALY at 24 months; (d) cost-effective analysis; (e) consumption of rescue medication; (f) individual patient placebo response; (g) blinding success; (h) treatment adherence; (i) AEs.

\section{Patient and public involvement}

People with KOA have been involved in the study design by participating in a focus group during study preparation and in a small pilot study. We conducted a prestudy patient focus group (three patient representatives involved) to discuss the study design, obtain their opinions and experience that are relevant to the study. We have also included two pilot participants (one in the active group and one in the placebo group) at each site (four in total) to assess the study procedures, the success of blinding, the burden of the intervention and time consumed in different processes in this research. The pilot participants received two injections (baseline and week 3) and was followed up to 1 month from the baseline visit. They reviewed participant-related documents and provided feedback on them. They will be asked to review the lay summary and an infographic summarising the main study results before sending them out to the study participants during study close-out. The data collected from the pilot participants will be excluded from the statistical analysis.

\section{Study procedures}

An outline of the study events and procedures is summarised in figure 1 and table 1 .

\section{Screening and radiographic assessment}

People who are interested in the study will be required to complete an online prescreening survey (http://tinyurl. $\mathrm{com} /$ sculptor-trial) to determine their initial eligibility. Potentially eligible participants will be redirected to 


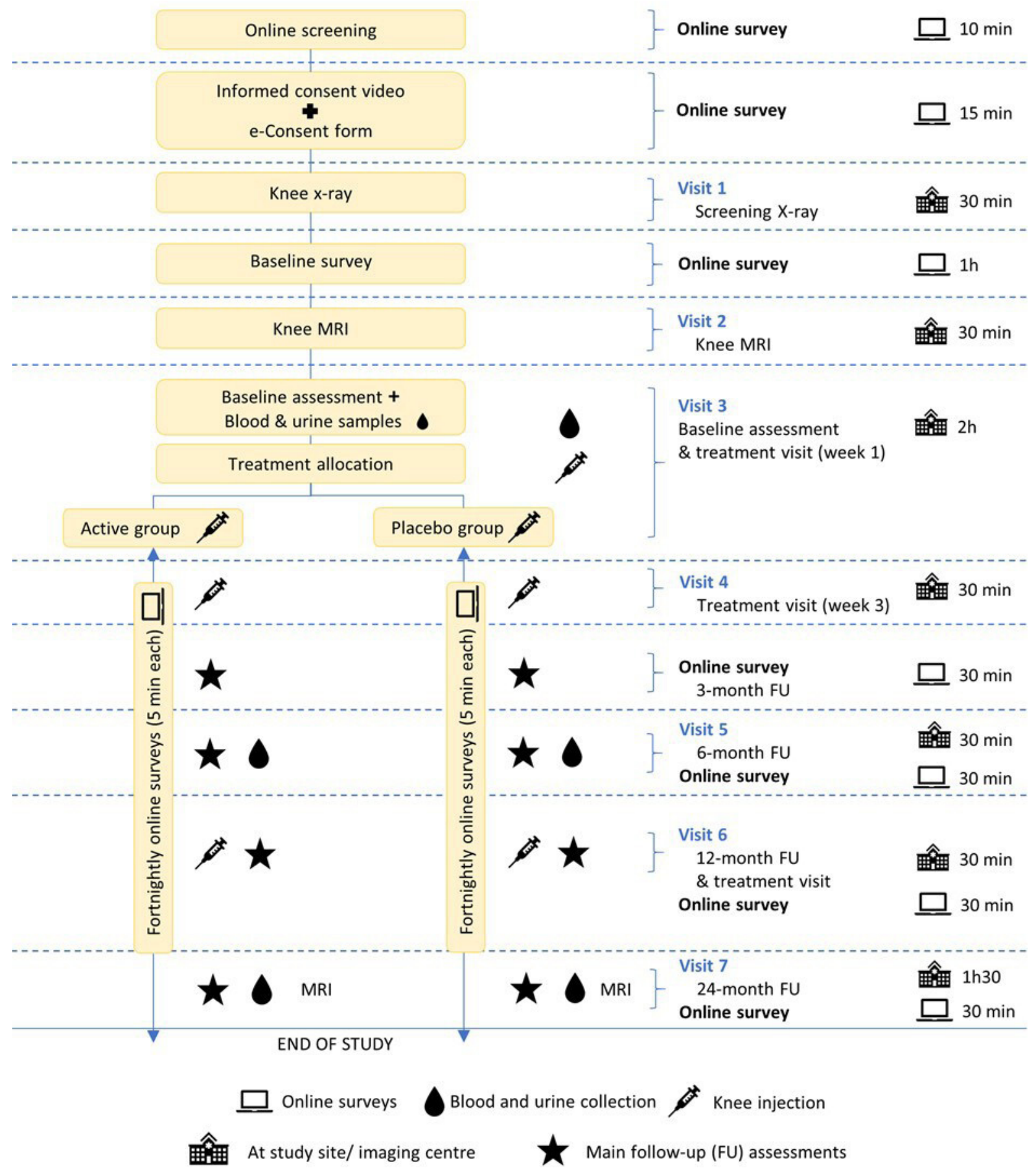

Figure 1 Flow diagram of the study protocol.

complete an electronic-Participant Consent Form (e-PCF) after watching a video containing further details about the study (online supplemental file 2 consent form). Individuals who sign the e-PCF will be referred to have a knee X-ray taken either at Castlereagh Imaging at St Leonards (Sydney, Australia) or at Qscan Radiology (North Hobart, Australia) (visit 1). Fixed-flexion PA knee radiographs for both knees will be taken using the Synaflexer X-ray positioning frame with feet externally rotated $10^{\circ}$, the knees and thighs touched the vertical platform anteriorly and the X-ray beam angulated $10^{\circ}$ (the angulation might be adjusted in order to achieve the best quality of films) caudally, ${ }^{39}$ which will be used to assess eligibility and exclude those who have predominant lateral tibiofemoral KOA. The skyline view of the study knee will be taken with $65^{\circ}$ of knee flexion, which will be used to exclude those who have predominant patellofemoral KOA. All these radiographs will be assessed by a trained rheumatologist.

Baseline survey (online)

After X-ray assessment, eligible participants who are taking any pain medications or analgesics will be requested to undergo a 1 week wash-out. After an appropriate wash-out if applicable, participants will be sent a pre-baseline 


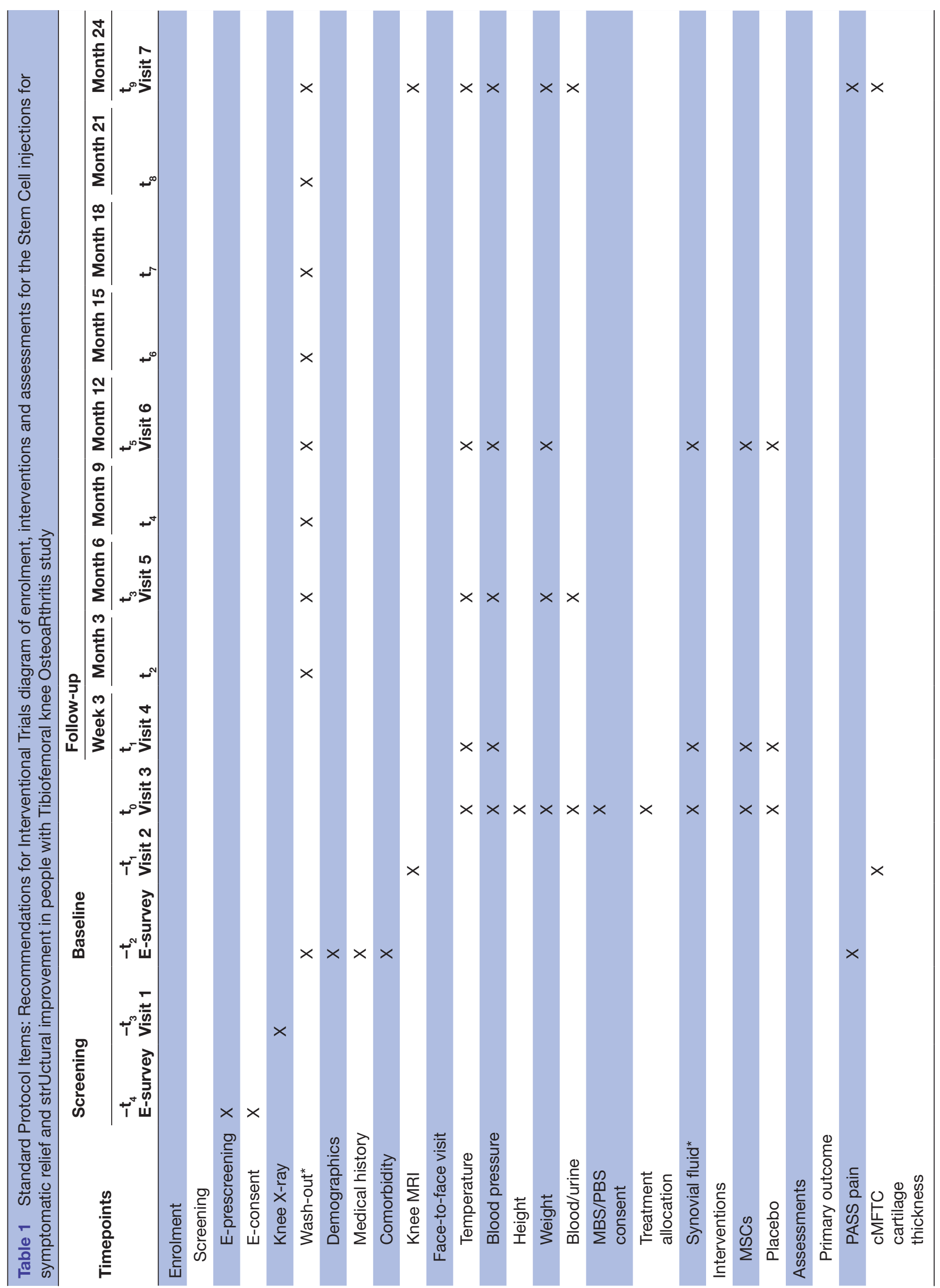




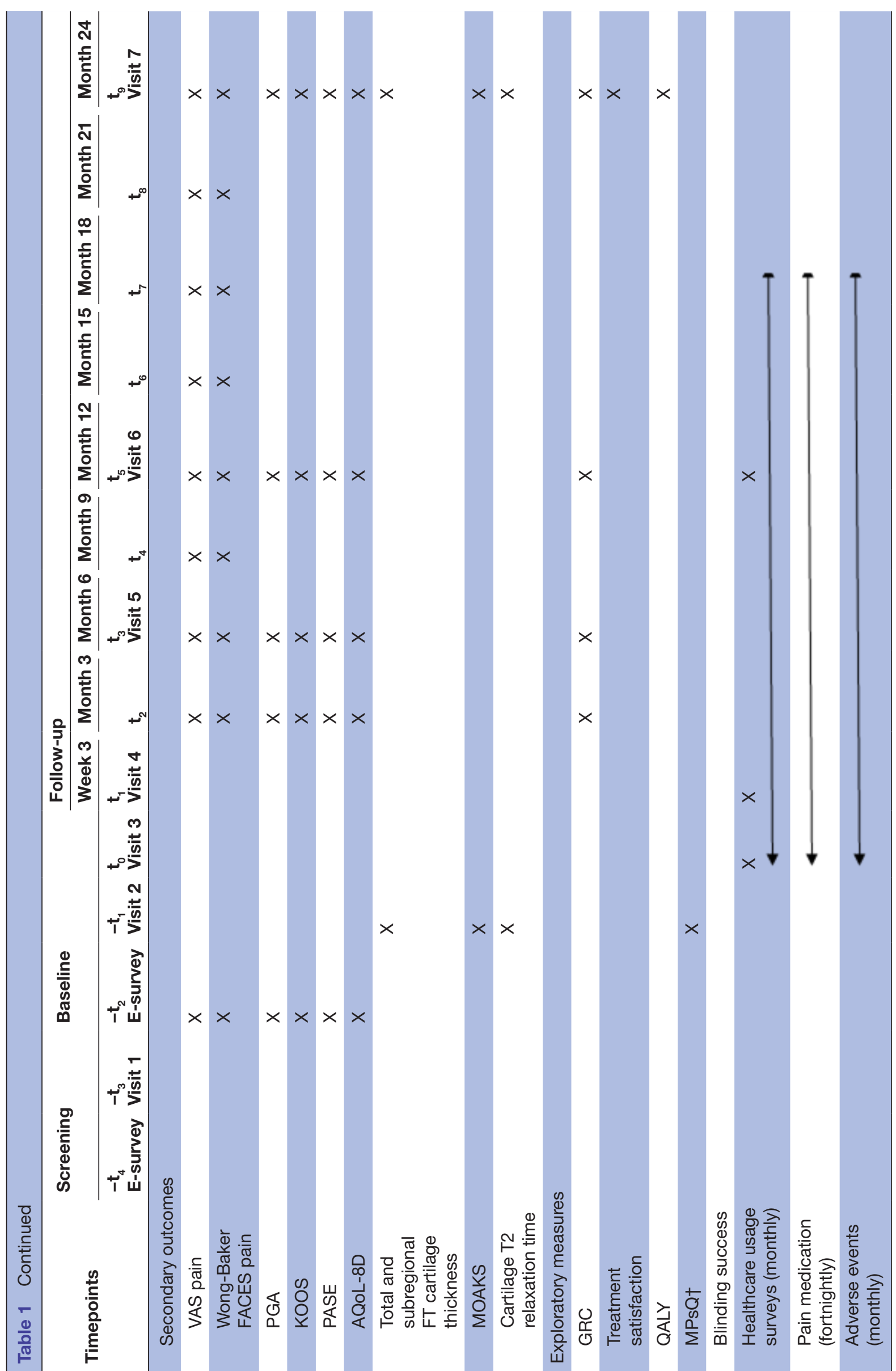

웜

官

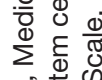

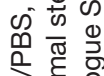

की त्र

बे बे ब

ठํำ

क जि

ह

䒓

里 원

든 은

तั $\frac{1}{2}$

कै 증

믄 음 흔

긍 व

至市

Ф

$\bar{x} \div \frac{1}{4}$

की Фै ह

․ㅡㅇ

बें

$\frac{\pi}{\pi} \sum^{\frac{\pi}{0}} \frac{0}{0}$

엉 은

눙문

बิ

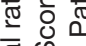

बृ

음 ఖ 임

ن

蓄菏

즈 ํㅡㄹ

웡 站

응

武 $\frac{0}{\Sigma}$

음

है

진 $\sum \frac{1}{1}$

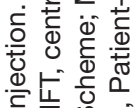

造 $\infty$

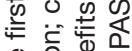

동 을

ब ब

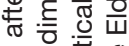

엉 농

엉 융

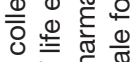

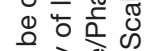

言吾

ब.

कृ 긍 $\sum_{1}^{\infty} \infty_{0}^{\infty}$

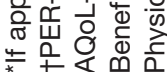


survey for further assessment of their knee pain intensity. Those who are eligible (ie, knee pain $\geq 40$ and $\leq 90$ on a 0-100 VAS) will continue to complete the baseline survey for the collection of baseline information including demographics, clinical characteristics, psychological and comorbidity assessments, and self-reported outcome measures.

\section{Knee MRI (visit 2)}

Participants who complete the baseline survey will be referred to have a knee MRI at either Castlereagh Imaging (Cremorne, Sydney) or Qscan Radiology (North Hobart). The details of the 3.0T MRI machines, dedicated knee coils and acquisition sequences at each site are specified in tables 2 and 3. Participants who have fractures, infections or tumours will be excluded after the MRI assessment.

\section{Baseline visit (visit 3) and enrolment}

Eligible participants after MRI will be required to attend a face-to-face baseline assessment at a designated site before their first injection. Temperature, blood pressure, height and weight will be measured during this visit (the injection will not proceed in case of systolic blood pressure over $180 \mathrm{~mm} \mathrm{Hg}$ ). MBS/PBS consent form for data extraction to be used in the cost-effectiveness analysis will be collected during this visit. Thirty millilitre of blood and $50 \mathrm{~mL}$ of urine will be collected for safety monitoring purposes, part of the collected samples will be stored for those who consent for deposition in the biobank for future analysis. The blood samples used for glucose (random), chemistry, liver function, full blood count, estimated glomerular filtration rate, $\mathrm{C}$ reactive protein tests will be analysed by Pathology North (Sydney) or Pathology South (Hobart). The urinalysis will be conducted by the research team using urine test strips, which will include specific gravity, $\mathrm{pH}$, leukocytes, nitrite, protein, glucose, ketones, urobilinogen, bilirubin, and blood.

After the completion of the face-to-face baseline assessment, participants will be considered to enter the study. A study enrolment number (composed of REDCap ID and enrolment order) will be assigned and recorded in all participant-specific study documents. Enrolled participants will receive a Participant's Identification Card for the purpose of urgent contact in case of any medical issues. A notification letter will be posted to their general practitioner (GP) to inform of their enrolment in the study.

\section{Intervention preparation and injection}

In Sydney, the injections will be prepared and delivered to participants at Castlereagh Imaging. A dry shipper will be used to transport the frozen cells between the Kolling Institute and Castlereagh. In Hobart, the cells will be prepared and injected at the Menzies Institute. The MSCs CryoBag will be thawed in a $37^{\circ} \mathrm{C}$ water bath. The unblinded researcher will wear appropriate personal protective equipment and transfer $5 \mathrm{~mL}$ stem cell solution

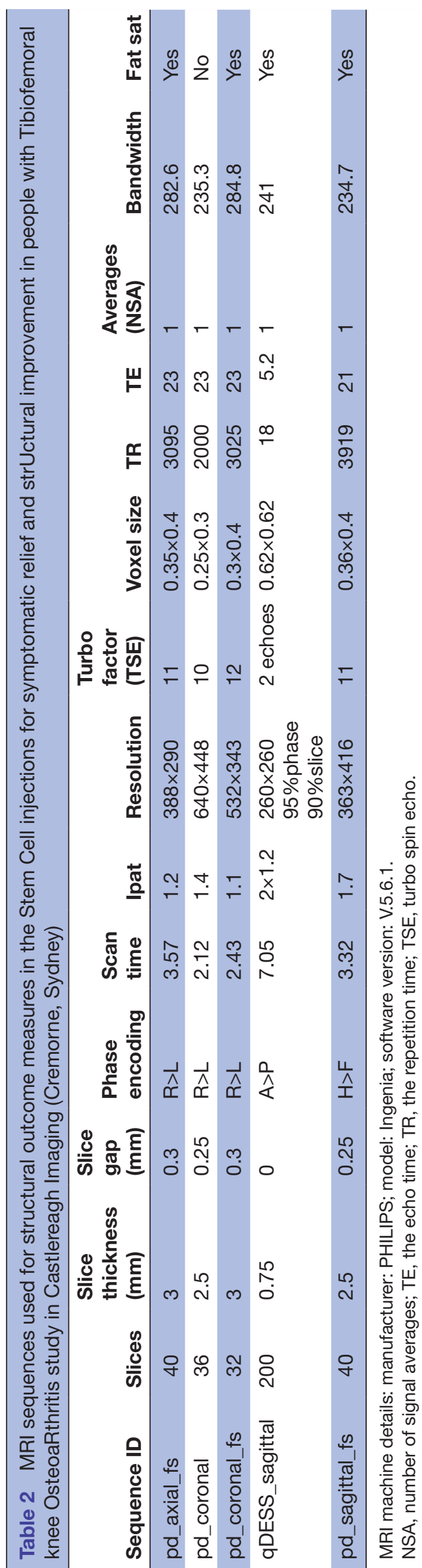




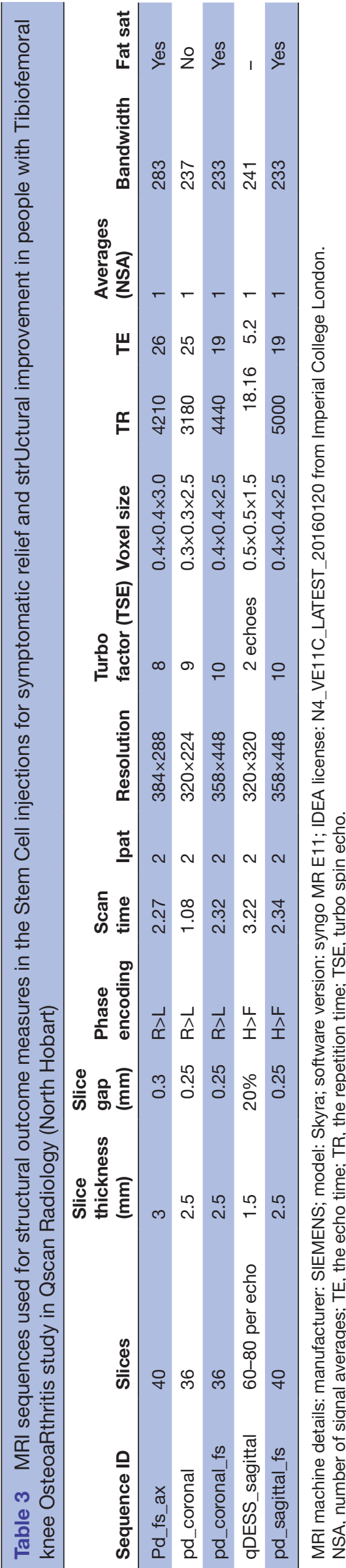

to a $5 \mathrm{~mL}$ syringe using an aseptic technique. The syringe will be covered with masking tape to occlude its contents and an amber connector will be used to mask the tip of the syringe. The same procedure will be used to prepare the placebo ( $5 \mathrm{~mL}$ normal saline). The sheathed/masked syringe will be injected within $60 \mathrm{~min}$ post-thaw. The administration procedure of MSCs and placebo will be the same with guidance by ultrasound imaging during a $10 \mathrm{~s}$ injection period into the knee joint. Participants will return to the same site for their second and third injection at week 3 (visit 4) and week 52 (visit 6) from the first injection.

In case of joint effusion, synovial fluid will be aspirated before the injection. If the synovial fluid appearance is abnormal (ie, cloudy, opaque and/or coloured), treatment injection will not proceed. The collected sample will be sent to pathology to check the cell count, culture and crystal analysis. If the results show a high white cell count, infection or crystal, then the participant will be withdrawn from the study and results will be sent to their GP for follow-up care. If the result is not clinically significant, the treatment injection will be rescheduled.

\section{Follow-up}

Participants will be asked to complete online follow-up assessment surveys at 3,6 (visit 5), 12 (visit 6) and 24 months (visit 7). Body temperature and blood pressure will be assessed at each visit after enrolment. Body weight will be measured at 6,12 and 24 months. Safety blood and urine samples will be collected at 6 and 24 months. A follow-up MRI scan will be performed at 24 months. Pain medication use will be monitored using a fortnightly online survey. Healthcare usage and AEs will be monitored using a monthly online survey. Pain intensity will be monitored every 3 months using an online survey.

\section{Adverse events}

Any untoward events that occur from the time of the enrolment will be monitored and assessed using a monthly survey indicating the start and end date of the event, details of the event, any actions taken and outcome. Participants will also be monitored for AEs at each study visit after the enrolment. The study physician will assess the severity (ie, severe, moderate, mild) and causality (ie, definitely related, probably related, possibly related, unlikely related, not related) of the AEs and give advice accordingly. Any abnormal blood and urine tests will be assessed as clinically significant or non-clinically significant.

An AE will be considered 'serious' when it causes: (a) death, (b) life-threatening event, (c) inpatient hospitalisation or prolongation of existing hospitalisation, (d) persistent or significant disability/incapacity, (e) a congenital anomaly/birth defect in the offspring of a participant.

Data and safety monitoring board (DSMB), composed of two clinical researchers, one clinician and one independent statistician, will meet every 6 months via 
videoconference and review the AEs. All serious AEs will be reported to DSMB within 24 hours of becoming aware of the events regardless of causality to ensure the safety of the participants.

\section{Participant retention and withdrawal}

Strategies to maximise retention will be implemented (eg, survey reminders, emails, phone calls, virtual birthday cards, gift vouchers, monthly health tips and survey reminders via SMS). A participant will be considered as a drop-out and will be excluded if he/she stops completing the surveys and is unable to be reached.

A participant will be able to withdraw from the study at any time by signing the electronic withdrawal form. In order to improve data retention, participants will have the option to continue to provide follow-up data via online surveys and to attend the final MRI visit, or not. If a participant chooses to stop their involvement completely, no further data will be collected from this participant, but previously collected data will be included in the analysis with the permission of the participant.

The investigator may also withdraw participants from the study to protect their safety. Participants who drop out and withdraw from the study will not be replaced.

\section{Statistical methods}

\section{Sample size estimation}

The sample size calculation was based on the primary objective to show statistical superiority of intra-articular MSCs injections compared with placebo for both coprimary outcomes PASS for knee pain and cMFTC cartilage thickness change at 24 months. Based on previous reports, the average proportion of patients who have achieved the PASS threshold was $35 \%$ in the placebo group (ranging from 33.1 to 35.5 ) and $48 \%$ in the intervention groups (ranging from $42.2 \%$ to $56.1 \%$ ). ${ }^{40}$ In a recent nested case-control study enrolling KOA patients, the change of cMFTC cartilage thickness over 24 months was $-0.32 \mathrm{~mm}(\mathrm{SD}=0.40)$ for those with narrowed medial tibiofemoral joint and persisted knee pain at baseline when compared with the control with neither radiographic nor pain progression $(-0.12 \mathrm{~mm}, \mathrm{SD}=0.28) .{ }^{42}$ A total number of 440 participants $(220$ per treatment arm) will provide $86 \%$ power to detect a $16 \%$ increase in the proportion of participants who achieve the PASS threshold (35\% vs $51 \%$ ) based on a two-sided significance level of $0.05 .{ }^{43}$ This sample size also provides $94 \%$ power to detect a between-group difference of $0.15 \mathrm{~mm}$ in mean cartilage thickness change over 24 months. ${ }^{44}$ The power to evaluate the joint effect of both coprimary endpoints is at least $80 \%$. The sample size allows for a drop-out rate of up to $20 \%$ over 24 months.

\section{Statistical analysis plan}

The statistical analysis will be performed by a qualified biostatistician who will be blinded to the group allocation. Efficacy analyses will be according to modified intention to treat, including participants with available outcome data based on their randomised treatment allocation, regardless of compliance. Safety analyses will be by treatment received, including participants who received at least one dose of study treatment. For the study to be declared a success both coprimary efficacy endpoints need to be significant at the two-sided $\alpha$ level of 0.05 in favour of intra-articular MSCs injections.

Demographic characteristics and baseline scores will be presented to assess the comparability of treatment groups at baseline. Participant characteristics will be summarised as mean (SD) for continuous variables or medians (quartiles) if the distribution is skewed. Counts with percentages will be presented for categorical variables.

All continuous primary and secondary outcome measures will be summarised as means (SD) at each time point of interest by treatment group. The betweengroup difference (with 95\% CI) in mean change from baseline will be presented and compared using independent samples t-test. For binary outcome variables differences in proportions will be presented with $95 \%$ CIs and compared using $\chi^{2}$ tests. The conditional binomial test will be used when expected cell counts are small. The Wilcoxon rank-sum test will be used to compare ordinal or discrete/continuous outcomes but not normally distributed, between groups. Poisson regression will be employed to model count variables.

Analysis adjusted for baseline values to account for possible floor and ceiling effects will be performed for the following outcome measures: PASS, VAS, WBS, PGA, KOOS, PASE, AQoL-8D and cartilage thickness (cMFTC and other femorotibial plates and subregions). For continuous outcomes analysis of covariance models will be fitted separately at each timepoint of interest with the change from baseline as the dependent variable. For binary outcomes logistic regression will be used. Other covariables of interest will include age, gender and body mass index. Generalised estimating equations will be used to explore trends in the effect of treatment over time.

To assist with the interpretation of the results, we will calculate the cut-point for the minimal clinically important improvement for pain using the mean change anchor-based approach. The pain item of GRC scale will be used as the anchoring question with participants answering 'slightly better' considered to be reporting a minimum clinically important improvement. ${ }^{45}$

The two-sided 5\% significance level will be used for all hypothesis tests, with no adjustments for multiple testing. No interim analysis will be carried out for this study.

\section{Cost-effectiveness analysis}

The measure of effectiveness will be QALYs based on measures obtained from the AQoL at each time point and transformed into a utility index using weights derived from the Australian population. Costs to the healthcare system will be based on MBS and PBS costs. Costs of hospitalisations will be valued at standard Australian Refined Diagnosis Related Group cost weights. Costs of the study treatments and private healthcare services will 
be valued at published standard rates, if available, or as reported by participants in their diaries. The aggregate of such costs will be used to estimate healthcare costs incurred by participants in both arms of the trial. An incremental cost-effectiveness ratio will be estimated based on the difference in costs and QALYs over 24 months between treatment arms with $95 \%$ CI calculated using non-parametric bootstrapping and presented as an acceptability (net benefits) curve for a range of willingness to pay for a QALY.

\section{Data management}

Electronic case report forms built in REDCap, a secure web-based application designed to support data capture for research studies and hosted on The University of Sydney server, will be used to facilitate the collection of the data throughout the study. The data will be stored in a re-identifiable format to ensure confidentiality. All computer-based files will be stored in OneDrive, which will be accessible only to the researchers. A back-up of REDCap data will be regularly stored in the University of Sydney Research Data Store. Self-monitoring of data entry will be used to maximise data quality.

\section{ETHICS AND DISSEMINATION}

This protocol has been approved by The University of Sydney (USYD) Human Research Ethics Committee (HREC) \#: 2020/119 and The University of Tasmania (UTAS) HREC \#: H0021868. All participants will be required to provide informed consent. Digital informed consent to participate will be obtained from all participants through the REDCap software. The results of this study will be disseminated through conferences, social media and scientific publications. No information which could lead to the identification of a participant will be included in the dissemination of results.

\section{Timelines}

The pilot study in Sydney commenced in November and completed in December 2020. The pilot study in Hobart commenced in May and completed in June 2021. Recruitment has commenced since January 2021 in Sydney and August 2021 in Hobart. The complete data collection is anticipated to be completed in June 2024. The study close-out is anticipated in December 2024.

\footnotetext{
Author affiliations

${ }^{1}$ Department of Rheumatology, Royal North Shore Hospital, Northern Clinical School, Faculty of Medicine and Health, The University of Sydney, Sydney, New South Wales, Australia

${ }^{2}$ Institute of Bone and Joint Research, Kolling Institute of Medical Research, The University of Sydney, Sydney, New South Wales, Australia

${ }^{3}$ Menzies Institute for Medical Research, University of Tasmania, Hobart, Tasmania, Australia

${ }^{4}$ Institute of Anatomy and Cell Biology and Ludwig Boltzmann Institute for Arthritis and Rehabilitation, Paracelsus Medical University Salzburg, Salzburg, Austria ${ }^{5}$ Chondrometrics GmbH, Freilassing, Germany
}

${ }^{6}$ Raymond Purves Bone and Joint Research Labs, Kolling Institute of Medical Research, Faculty of Medicine and Health, The University of Sydney, Sydney, New South Wales, Australia

${ }^{7}$ Castlereagh Imaging, St Leonards, Sydney, New South Wales, Australia

${ }^{8}$ Centre for Health Economics, Monash University, Clayton, Victoria, Australia

${ }^{9}$ School of Medicine, College of Health and Medicine, University of Tasmania Faculty of Health, Hobart, Tasmania, Australia

${ }^{10} \mathrm{NHMRC}$ Clinical Trials Centre, The University of Sydney, Sydney, New South Wales, Australia

${ }^{11}$ Representative of People with Knee 0steoarthritis, Sydney, New South Wales, Australia

${ }^{12}$ Department of Clinical Sciences Lund, Orthopaedics, Lund University, Lund, Sweden

${ }^{13}$ Clinical Research Centre, Zhujiang Hospital, Southern Medical University, Guangzhou, Guangdong, China

\section{Twitter Leticia A Deveza @LeticiaDeveza and David J Hunter @ProfDavidHunter}

Acknowledgements We thank patient representatives and pilot participants who were involved in the study design for their valuable feedback on the study procedures and relevant documents. Cynata Therapeutics Ltd (Carlton, Victoria, Australia) provided the investigational products free of cost. Cynata provided feedback on the study design but they will have no role in the data analysis, interpretation, preparation and submission of the manuscript.

Contributors DJH is the grant holder and conceived of the study. $\mathrm{XL}$ drafted the paper. DJH, CD, SL, RO'C, SR and XW contributed to the study design. All investigators including $L A D, W M 0, K C, B A, F E, W W, C L, J L, A H, D H, ~ G H, T B$ revised this protocol critically for important intellectual content. SR, KS, KC, BA and SV will be responsible for the implementation.

Funding The Sculptor study is funded by an NHMRC Programme Grant (ID: APP1162874).

Competing interests DJH is supported by an NHMRC Investigator Grant and provides consulting advice for Merck Serono, TLC Bio, Tissuegene and Pfizer. CL has provided consulting advice for Merck Serono and Galapagos Pharmaceuticals, and receives research funding from numerous pharmaceutical companies through specific services/testing contract research agreements between and managed by The University of Sydney or the NSLHD. SL provides consulting advice for Arthro Therapeutics, Pfizer and Synartro. LAD has received partial reimbursement of a conference registration by Pfizer. FE is CEO/CMO and co-owner of Chondrometrics $\mathrm{GmbH}$, and he has provided consulting services to Merck KGaA, Abbvie, Samumed, Kolon-Tissuegene, Servier, Galapagos, Roche, Novartis, ICM and HealthLink. WW is co-owner of Chondrometrics $\mathrm{GmbH}$, and has provided consulting services to Galapagos.

Patient consent for publication Consent obtained directly from patient(s).

Provenance and peer review Not commissioned; externally peer reviewed.

Supplemental material This content has been supplied by the author(s). It has not been vetted by BMJ Publishing Group Limited (BMJ) and may not have been peer-reviewed. Any opinions or recommendations discussed are solely those of the author(s) and are not endorsed by BMJ. BMJ disclaims all liability and responsibility arising from any reliance placed on the content. Where the content includes any translated material, BMJ does not warrant the accuracy and reliability of the translations (including but not limited to local regulations, clinical guidelines, terminology, drug names and drug dosages), and is not responsible for any error and/or omissions arising from translation and adaptation or otherwise.

Open access This is an open access article distributed in accordance with the Creative Commons Attribution Non Commercial (CC BY-NC 4.0) license, which permits others to distribute, remix, adapt, build upon this work noncommercially, and license their derivative works on different terms, provided the original work is properly cited, appropriate credit is given, any changes made indicated, and the use is non-commercial. See: http://creativecommons.org/ licenses/by-nc/4.0/.

\section{ORCID iDs}

Xiaogian Liu http://orcid.org/0000-0002-6375-1800

Xia Wang http://orcid.org/0000-0003-3172-9390

Anthony Harris http://orcid.org/0000-0003-1641-3320

Stefan Lohmander http://orcid.org/0000-0002-5424-9448

David J Hunter http://orcid.org/0000-0003-3197-752X 


\section{REFERENCES}

1 Glyn-Jones S, Palmer AJR, Agricola R, et al. Osteoarthritis. Lancet 2015;386:376-87.

2 Safiri S, Kolahi A-A, Smith E, et al. Global, regional and national burden of osteoarthritis 1990-2017: a systematic analysis of the global burden of disease study 2017. Ann Rheum Dis 2020;79:819-28.

3 Sharma L. Osteoarthritis of the knee. N Engl J Med 2021;384:51-9.

4 Hunter DJ, Schofield D, Callander E. The individual and socioeconomic impact of osteoarthritis. Nat Rev Rheumatol 2014;10:437-41.

5 Wallace IJ, Worthington S, Felson DT, et al. Knee osteoarthritis has doubled in prevalence since the mid-20th century. Proc Natl Acad Sci U S A 2017;114:9332-6.

6 Cross M, Smith E, Hoy D, et al. The global burden of hip and knee osteoarthritis: estimates from the global burden of disease 2010 study. Ann Rheum Dis 2014;73:1323-30.

7 Chen F, Su W, Bedenbaugh AV, et al. Health care resource utilization and burden of disease in a U.S. Medicare population with a principal diagnosis of osteoarthritis of the knee. J Med Econ 2020;23:1151-8.

8 Wilson $\mathrm{R}$, Abbott $\mathrm{JH}$. The projected burden of knee osteoarthritis in New Zealand: healthcare expenditure and total joint replacement provision. N Z Med J 2019;132:53-65.

9 Bannuru RR, Schmid CH, Kent DM, et al. Comparative effectiveness of pharmacologic interventions for knee osteoarthritis: a systematic review and network meta-analysis. Ann Intern Med 2015;162:46-54.

10 Machado GC, Maher CG, Ferreira PH, et al. Efficacy and safety of paracetamol for spinal pain and osteoarthritis: systematic review and meta-analysis of randomised placebo controlled trials. BMJ 2015;350:h1225.

11 Hunter DJ, Bierma-Zeinstra S. Osteoarthritis. Lancet 2019;393:1745-59.

12 Pas HI, Winters M, Haisma HJ, et al. Stem cell injections in knee osteoarthritis: a systematic review of the literature. Br J Sports Med 2017:51:1125-33.

13 Piuzzi NS, Ng M, Chughtai M, et al. The stem-cell market for the treatment of knee osteoarthritis: a patient perspective. J Knee Surg 2018;31:551-6.

14 Colombini A, Perucca Orfei C, Kouroupis D, et al. Mesenchymal stem cells in the treatment of articular cartilage degeneration: new biological insights for an old-timer cell. Cytotherapy 2019;21:1179-97.

15 Song Y, Du H, Dai C, et al. Human adipose-derived mesenchymal stem cells for osteoarthritis: a pilot study with long-term follow-up and repeated injections. Regen Med 2018;13:295-307.

16 Freitag J, Bates D, Wickham J, et al. Adipose-derived mesenchymal stem cell therapy in the treatment of knee osteoarthritis: a randomized controlled trial. Regen Med 2019;14:213-30.

17 Matas J, Orrego M, Amenabar D, et al. Umbilical cord-derived mesenchymal stromal cells (MscS) for knee osteoarthritis: repeated MSC dosing is superior to a single MSC dose and to hyaluronic acid in a controlled randomized phase I/II trial. Stem Cells Trans/ Med 2019;8:215-24.

18 Lamo-Espinosa JM, Blanco JF, Sánchez M, et al. Phase II multicenter randomized controlled clinical trial on the efficacy of intra-articular injection of autologous bone marrow mesenchymal stem cells with platelet rich plasma for the treatment of knee osteoarthritis. $J$ Trans/ Med 2020;18:356.

19 Emadedin M, Labibzadeh N, Liastani MG, et al. Intra-articular implantation of autologous bone marrow-derived mesenchymal stromal cells to treat knee osteoarthritis: a randomized, tripleblind, placebo-controlled phase $1 / 2$ clinical trial. Cytotherapy 2018;20:1238-46.

20 Mushahary D, Spittler A, Kasper C, et al. Isolation, cultivation, and characterization of human mesenchymal stem cells. Cytometry $A$ 2018;93:19-31.

21 Yu SP, Hunter DJ. Intra-articular therapies for osteoarthritis. Expert Opin Pharmacother 2016;17:2057-71.

22 Lopa S, Colombini A, Moretti M, et al. Injective mesenchymal stem cell-based treatments for knee osteoarthritis: from mechanisms of action to current clinical evidences. Knee Surg Sports Traumatol Arthrosc 2019;27:2003-20.

23 Ozay El, Vijayaraghavan J, Gonzalez-Perez G, et al. Cymerus ${ }^{\mathrm{TM}}$ iPSC-MSCs significantly prolong survival in a pre-clinical, humanized mouse model of graft-vs-host disease. Stem Cell Res 2019;35:101401.
24 Dominici M, Le Blanc K, Mueller I, et al. Minimal criteria for defining multipotent mesenchymal stromal cells. The International Society for cellular therapy position statement. Cytotherapy 2006;8:315-7.

25 Bloor AJC, Patel A, Griffin JE, et al. Production, safety and efficacy of iPSC-derived mesenchymal stromal cells in acute steroid-resistant graft versus host disease: a phase I, multicenter, open-label, doseescalation study. Nat Med 2020;26:1720-5.

26 Thavapalachandran S, Le TYL, Romanazzo S, Rashid FN, et al. Pluripotent stem cell-derived mesenchymal stromal cells improve cardiac function and vascularity after myocardial infarction. Cytotherapy 2021. doi:10.1016/j.jcyt.2021.07.016. [Epub ahead of print: 26 Sep 2021].

27 Altman R, Asch E, Bloch D, et al. Development of criteria for the classification and reporting of osteoarthritis: classification of osteoarthritis of the knee. Arthritis \& Rheumatism 1986;29:1039-49.

28 Tubach F, Ravaud P, Baron G, et al. Evaluation of clinically relevant states in patient reported outcomes in knee and hip osteoarthritis: the patient acceptable symptom state. Ann Rheum Dis 2005;64:34-7.

29 Wirth W, Hellio Le Graverand M-P, Wyman BT, et al. Regional analysis of femorotibial cartilage loss in a subsample from the osteoarthritis initiative progression subcohort. Osteoarthritis Cartilage 2009:17:291-7.

30 Lai Y-F, Lin P-C, Chen C-H, et al. Current status and changes in pain and activities of daily living in elderly patients with osteoarthritis before and after unilateral total knee replacement surgery. J Clin Med 2019;8. doi:10.3390/jcm8020221. [Epub ahead of print: 0802 2019].

31 Roos EM, Lohmander LS. The knee injury and osteoarthritis outcome score (KOOS): from joint injury to osteoarthritis. Health Qual Life Outcomes 2003;1:64.

32 Martin KA, Rejeski WJ, Miller ME, et al. Validation of the PASE in older adults with knee pain and physical disability. Med Sci Sports Exerc 1999;31:627-33.

33 Richardson J, lezzi A, Khan MA, et al. Validity and reliability of the assessment of quality of life (AQoL)-8D multi-attribute utility instrument. Patient 2014;7:85-96.

34 Hunter DJ, Guermazi A, Lo GH, et al. Evolution of semi-quantitative whole joint assessment of knee oa: MOAKS (MRI osteoarthritis knee score). Osteoarthritis Cartilage 2011;19:990-1002.

35 Kamper SJ, Maher CG, Mackay G. Global rating of change scales: a review of strengths and weaknesses and considerations for design. $J$ Man Manip Ther 2009;17:163-70.

36 Cleland J, Hutchinson C, Khadka J, et al. A review of the development and application of generic Preference-Based instruments with the older population. Appl Health Econ Health Policy 2019;17:781-801.

37 Pereira A, Gossuin C, Helleputte T. Method for prediction of a placebo response in an individual, 2017. Available: Patent US 15/308,502; US2017/0053082

38 Pereira A, Duale C, Clermont F, et al. (171) characterization and prediction of placebo responders in peripheral neuropathic patients in a 4-week analgesic clinical trial. J Pain 2016;17:S18.

39 Charles HC, Kraus VB, Ainslie M, et al. Optimization of the fixedflexion knee radiograph. Osteoarthritis Cartilage 2007;15:1221-4.

40 Hochberg MC, Wohlreich M, Gaynor P, et al. Clinically relevant outcomes based on analysis of pooled data from 2 trials of duloxetine in patients with knee osteoarthritis. $J$ Rheumatol 2012;39:352-8.

41 Dougados M, Moore A, Yu S, et al. Evaluation of the patient acceptable symptom state in a pooled analysis of two multicentre, randomised, double-blind, placebo-controlled studies evaluating lumiracoxib and celecoxib in patients with osteoarthritis. Arthritis Res Ther 2007;9:R11.

42 Eckstein F, Collins JE, Nevitt MC, et al. Brief report: cartilage thickness change as an imaging biomarker of knee osteoarthritis progression: data from the foundation for the National Institutes of health osteoarthritis biomarkers Consortium. Arthritis Rheumatol 2015;67:3184-9.

43 Tubach F, Ravaud P, Beaton D, et al. Minimal clinically important improvement and patient acceptable symptom state for subjective outcome measures in rheumatic disorders. J Rheumatol 2007;34:1188-93.

44 Hamasaki T, Evans SR, Asakura K. Design, data monitoring, and analysis of clinical trials with co-primary endpoints: a review. J Biopharm Stat 2018;28:28-51.

45 Copay AG, Subach BR, Glassman SD, et al. Understanding the minimum clinically important difference: a review of concepts and methods. Spine J 2007;7:541-6. 